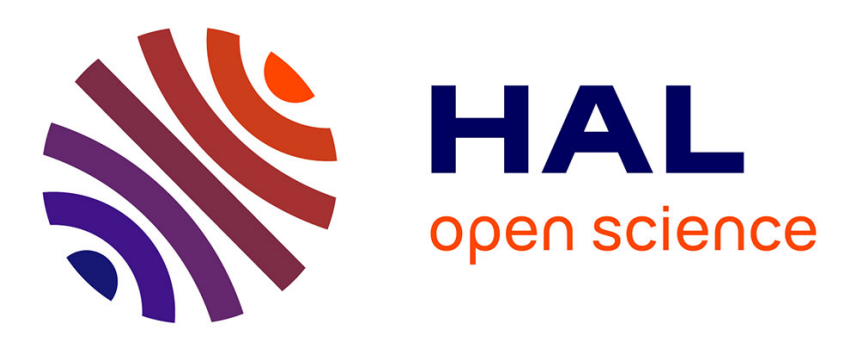

\title{
Molecular nanocrystals grown in sol-gel thin films for ultrabright chemical sensor applications
}

\author{
Virginie Monnier, Nicolas Sanz, M. Bacia, R. Pansu, A. Ibanez
}

\section{To cite this version:}

Virginie Monnier, Nicolas Sanz, M. Bacia, R. Pansu, A. Ibanez. Molecular nanocrystals grown in solgel thin films for ultrabright chemical sensor applications. Photonics Europe, Apr 2006, Strasbourg, France. 10.1117/12.662949 . hal-01846288

\section{HAL Id: hal-01846288 \\ https://hal.science/hal-01846288}

Submitted on 23 Jul 2018

HAL is a multi-disciplinary open access archive for the deposit and dissemination of scientific research documents, whether they are published or not. The documents may come from teaching and research institutions in France or abroad, or from public or private research centers.
L'archive ouverte pluridisciplinaire HAL, est destinée au dépôt et à la diffusion de documents scientifiques de niveau recherche, publiés ou non, émanant des établissements d'enseignement et de recherche français ou étrangers, des laboratoires publics ou privés. 


\title{
Molecular nanocrystals grown in sol-gel thin films for ultrabright chemical sensor applications
}

\author{
V. Monnier * a , N. Sanz ${ }^{\text {a }}$, M. Bacia ${ }^{\text {a }}$, R. Pansu ${ }^{\text {b }}$, A. Ibanez ${ }^{\text {a }}$ \\ ${ }^{a}$ Laboratoire de Cristallographie UPR 5031, associé à l'Université Joseph Fourier et à l'Institut \\ National Polytechnique de Grenoble, CNRS Grenoble BP166, 25 Avenue des Martyrs, 38042 \\ Grenoble Cedex 09, FRANCE \\ ${ }^{\mathrm{b}}$ Laboratoire de Photophysique et Photochimie Supramoléculaire et Macromoléculaire UMR 8531, \\ ENS de Cachan, CNRS, 61 Avenue du Président Wilson, 94235 Cachan Cedex, FRANCE
}

\begin{abstract}
Polyaromatic dyes were used to synthesize molecular nanocrystals in sol-gel thin films for sensor applications. Fluorescence Confocal Microscopy (FCM) and Transmission Electron Microscopy (TEM) experiments showed the advantages of our nanocrystallization process compared with microcrystallization in free solutions. Indeed, we obtained well-dispersed and spherical nanocrystals with a narrow size distribution, exhibiting a good crystallinity. Time-resolved fluorescence spectroscopy allowed us to measure fluorescence lifetimes of nanocrystals in presence of molecular probes. Then, chemical sensoring properties of these molecular nanocrystals were demonstrated.
\end{abstract}

Keywords : nanocrystals, sol-gel, nanosensors, thin films, fluorescence.

\section{INTRODUCTION}

Recently, we have engineered new hybrid organic-inorganic materials through a simple and generic preparation of stable organic nanocrystals grown in sol-gel thin films prepared by spin coating [1-3]. This process is based on the confined nucleation and growth of dyes in the pores of sol-gel networks. These nanocomposite coatings not only combine the optical properties of organic phases (luminescence, NLO properties, photochromism, ...) with those of amorphous inorganic materials (high stability, convenient processing and shaping) but also the advantages of nanocrystals: preparation of low diffusing samples for visible or IR laser beams and higher stability than dispersed molecules. The aim of this work is to design a new type of ultrabright fluorescent nanosensors, with sensitivity 100 to 1000 times than existing fluorescent nanosensors. This will allow the detection of small quantities of molecular probes without needing its amplification. Indeed, organic nanocrystals exhibit higher photostability and luminosity than isolated molecules dispersed in solutions or in solid matrices such as sol-gel or polymers. The fluorescence intensity is also significantly enhanced because a $500 \mathrm{~nm}$-diameter nanocrystal is an aggregation of around $10^{6}-10^{7}$ fluorescent molecules. Moreover, the nanoparticles crystallinity favors the delocalization of the initial excitation in the nanocrystal, which thus behaves like a single fluorescent transmitter. Furthermore, the open porosity of the sol-gel coatings can be modulated from around one nanometer (microporosity) to several tenths of nanometers (mesoporosity) by using different type of sol-gel precursors. This porosity adjustment at the nanometer-scale would allow protecting these nanosensors against unwanted interactions, the silicate matrix playing thus the role of a filter in size, charge and polarity. In this paper, we firstly present the preparation of our samples: microcrystals obtained from free solutions spread out onto substrates (free microcrystals) and nanocrystals grown in sol-gel thin films. Free microcrystals were prepared to select the best chromophore for sensor application and to underline the advantages of our method of nanocrystallization in sol-gel matrices. Secondly, we present the nanocrystals characterizations performed by fluorescence confocal microscopy (FCM) and transmission electron microscopy (TEM). Then, we report electronic diffraction results obtained both for microcrystals from free solutions spread out onto substrates (free microcrystals) and for nanocrystals grown in sol-gel thin films. Finally, we present chemical sensor properties of the nanocrystals, evidenced by time-resolved fluorescence spectroscopy.

Organic Optoelectronics and Photonics II, edited by Paul L. Heremans, Michele Muccini, Eric A. Meulenkamp, Proc. of SPIE Vol. 6192, 61921I, (2006) · 0277-786X/06/\$15 · doi: 10.1117/12.662949 


\section{PREPARATION OF FREE MICROCRYSTALS AND NANOCRYSTALS IN SOL-GEL THIN FILMS}

We selected two polyaromatic dyes, tetracene (2, 3-benzanthracene) and rubrene $(5,6,11,12$-tetraphenylnaphthacene) which exhibit high fluorescence efficiency in the crystal state. To prepare free microcrystals, we dissolved organic powder of tetracene in tetrahydrofuran (THF) at the concentration $\mathrm{C}=1.1 \mathrm{~g} / \mathrm{L}$, then the solution was deposited on a microscope glass slide by spin-coating with a rotation speed of $4000 \mathrm{rpm}$. Rapid evaporation of the solvent at the end of rotation induced nucleation and growth of microcrystals on the substrate.

Nanocrystals in sol-gel thin films were also prepared by spin-coating. We started from solutions containing the solvent, the dye, silicon alkoxides and water, which are inserted in airtight flasks. In this work, solutions were obtained from a mixture of two alkoxide precursors: 1 TMSE (1,2-bis(trimethylsilyl)ethane) [4] for 2 TMOS (tetramethoxysilane). The sols were synthesized under acid $(\mathrm{HCl}, \mathrm{pH} \approx 1)$ catalyzed conditions by one step hydrolysis and condensation of the alkoxide precursors during $18 \mathrm{~h}$ at $80^{\circ} \mathrm{C}$ with one water molecule per -OR function $\left(\mathrm{h}=\left[\mathrm{H}_{2} \mathrm{O}\right] /[-\mathrm{OR}]=1\right)$. THF was used to dissolve the organic dyes and to mix water of hydrolysis with alkoxide precursors; the molar ratio solvent / alkoxide, s, was set from 5 to 10 for all the thin films studied here. The heating at $80^{\circ} \mathrm{C}$ allows to rapidly dissolve the organic powder and to enhance the hydrolysis and condensation kinetics leading to the formation of silicate chains dispersed in the solution. This sol ageing was necessary to control the sol viscosity, around $10-20 \mathrm{cps}$, to obtain high quality films, to ensure the control the coating thickness and the particle growth and to avoid the nanocrystal coalescence. The resulting sols, which were stable during several weeks, are deposited at room temperature by spincoating onto thin microscope slides with a rotation speed of $4000 \mathrm{rpm}$. Thus, films of around $0.5 \mu \mathrm{m}$ thick have been prepared. These nanocomposite coatings were then stabilized by annealings at $100^{\circ} \mathrm{C}$. The dye concentration, expressed as the molar ratio $\mathrm{d}=$ organic / alkoxide $\approx 5.10^{-4}-10^{-3}$, depends on the dye solubility in THF, the nature of the host matrix and on the wanted particle sizes. The later was targeted here between 150 and $500 \mathrm{~nm}$ in diameter to favor the nanocrystal observations and characterizations by fluorescence confocal microscopy.

\section{MICROSCOPIC CHARACTERIZATIONS}

We have first visualized the organic crystals through fluorescence confocal microscopy (FCM) excited by the $488 \mathrm{~nm}$ beam of a $15 \mathrm{~mW}$ Argon laser. The confocal laser scanning microscope is a LSM 510 from Zeiss. A comparison of crystallization obtained without sol-gel matrix (free microcrystals) and nanocrystallization with sol-gel matrix was done to underline the particularities of our nanocrystallization process. Images obtained by FCM are presented on Figure 1.

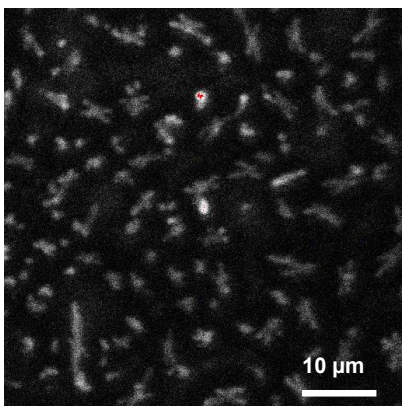

$1 \mathrm{a}$

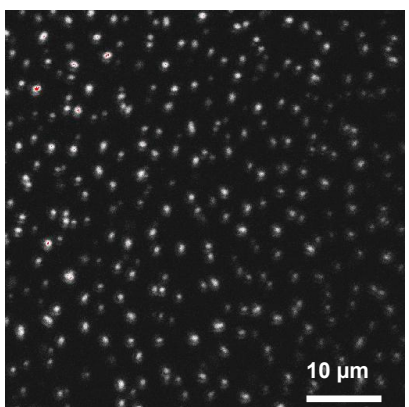

$1 \mathrm{~b}$

Figure 1 : Free microcrystals of tetracene (a) and tetracene nanocrystals embedded in sol-gel thin films (b) visualized by FCM

In Figure 1a, free microcrystals of tetracene have a stick shape and present a large size distribution from 0.5 to $5 \mu \mathrm{m}$. On the other hand, Figure 1b diplays nanocrystals of tetracene grown in sol-gel thin film, which are well-dispersed and embedded in the silicate coating, with a size distribution around $600 \mathrm{~nm}$. Rubrene nanocrystals in sol-gel thin film were 
also observed for several dye concentrations $\mathrm{d}=$ organic / alcoxide, inside the same sol-gel matrix TMSE +2 TMOS. FCM images of these samples are presented in Figure 2.

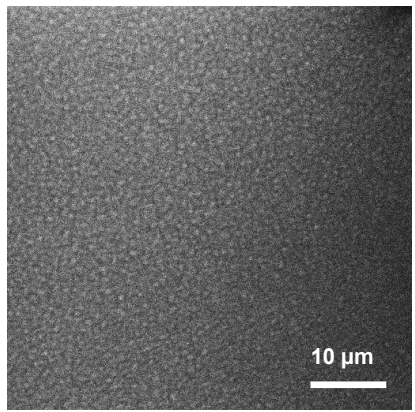

$2 a$

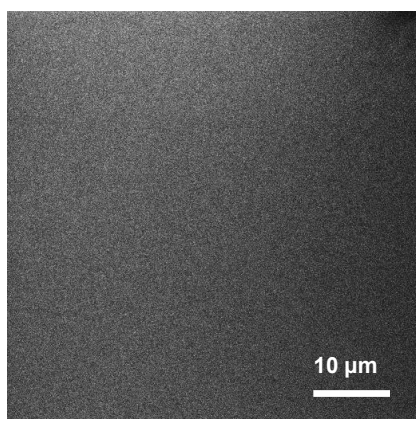

$2 \mathrm{~b}$

Figure 2 : Nanocrystals of rubrene prepared with $d=10^{-3}$ (a) and $d=5.10^{-4}$ (b) observed by FCM.

Figures $2 \mathrm{a}$ and $2 \mathrm{~b}$ show a high concentration of rubrene nanocrystals in the sol-gel thin film. The average size of the nanocrystals is around $300 \mathrm{~nm}$ for $\mathrm{d}=10^{-3}$ and $150 \mathrm{~nm}$ for $\mathrm{d}=5.10^{-4}$. Figure $2 \mathrm{~b}$ illustrates also the limit of resolution of FCM, around $200 \mathrm{~nm}$ in the $\mathrm{x}-\mathrm{y}$ plane. But even if characterizations are harder for nanocrystals of diameter lower than $200 \mathrm{~nm}$, this size is the one required for sensor applications. Indeed, spectroscopy results presented in section 5 will show that the detection is more sensitive with nanocrystals of $150 \mathrm{~nm}$ than $300 \mathrm{~nm}$.

As we were limited in FCM by the weak resolution of this technique, we also performed images by transmission electron microscopy (TEM). Transmission Electron Microscopy micrographs and diffraction patterns were recorded with a Philips CM300 operated at $300 \mathrm{kV}$. Image acquisition was made with CCD Gatan 794 camera (DigitalMicrograph (Gatan) and digital-video data-acquisition software. In this case, the coatings are deposited onto cleaved crystal plates of sodium chloride. These substrates are dissolved in water and the films are then placed on a copper grid for TEM characterizations. Thus, one can observe well-defined molecular crystals with a spherical shape and narrow size distribution, ranging for example between 400 and $650 \mathrm{~nm}$ in the sample displayed in Figure 3.

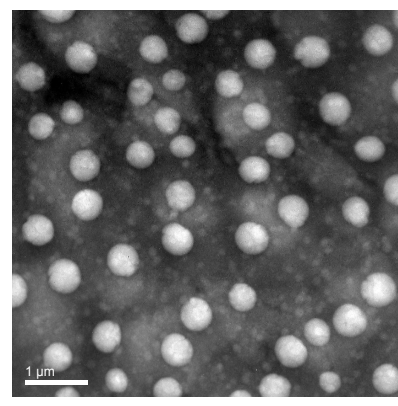

$3 a$

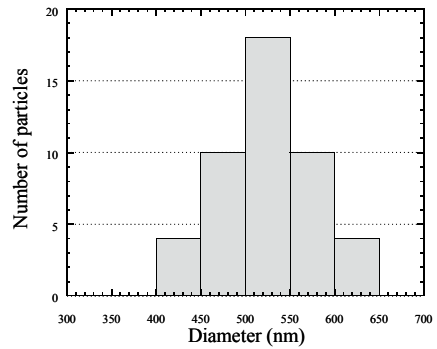

$3 b$

Figure 3 : TEM image of rubrene nanocrystals in sol-gel thin film (a) and corresponding histogram (b) showing a size distribution between 400 and $650 \mathrm{~nm}$.

In these imaging experiments carried out on microcrystals and nanocrystals we have illustrated the typical differences of nucleation and growth occurring in free solutions and in gel matrices. Indeed, in free solutions, in order to reduce the size and size distribution of tetracene crystals, we have applied a very high supersaturation through the rapid evaporation of THF solvent. This induces the nucleation of a high number of nuclei. This burst of nucleation leads to a fast decrease of organic molecules supersaturation in solution and to the end of nucleation step. Thus, according to the basic principles established in 1950 by V. K. La Mer [5], the nucleation is confined in time and allows to prepare small crystals of a few micrometers as shown in Figure 1a. Nevertheless, even if the nucleation occurs during a short period of time, the growth rate is not negligible during this step. This coupling between nucleation and growth explains the significant growth of the crystals and their wide size distribution (several micrometers). On the other hand, when the nucleation and growth of tetracene crystal is performed in sol-gel thin film the nucleation confinement in time is 
associated to a confinement in space of the nuclei in the pores of the sol-gel matrix previously polycondensed onto the substrate [6]. This additional spatial confinement reduces significantly the growth of the crystal which is diffusion controlled and the coupling effect between the nucleation and growth mechanisms. As the growth becomes negligible, the growth differences versus crystal orientations are not sufficient for the preparation of faceted crystals. Therefore, spherical nanocrystals, as shown in Figure 3a, are obtained. In addition, the gel network prevents the coalescence and aggregation of nanocrystals.

Figure 2 shows that the size of the nanocrystals is directly linked to the concentration d. Indeed, when $\mathrm{d}$ increases, the surpersaturation applied also increases, so the mechanisms of nucleation and growth are faster than polycondensation of silicate matrix. Thus, nanocrystals can grow during a delay longer so they present a larger size. Furthermore, the sursaturation is proportional to the growth speed, so the particles size is all the more enhanced.

Thus, with our nanocrystallization method, well-dispersed and stabilized spherical nanocrystals in a silicate porous matrix can be obtained with a narrow size distribution. These properties are crucial for the development of chemical sensors from these nanocomposite materials. Another important point is the crystallinity of the organic nanoparticles to obtain luminescence properties similar to that of a single molecule through the delocalization of the initial excitation in the whole nanocrystal; this leading to a single fluorescent transmitter behavior of the nanocrystal.

\section{STRUCTURAL CHARACTERIZATIONS}

We need to get structural information of these very small aggregates in order to find correlations between their crystallinity and their luminescence spectroscopy. Previously, differential scanning calorimetry experiments allowed evidencing clearly the melting points of organic nanocrystals grown in bulk sol-gel matrices. This demonstrated that these organic particles were crystallized [7]. Luminescence and absorption spectroscopies were also used to identify different polymorphs nanocrystallized in bulk gel glasses [8]. Then, the crystallinity of non-centrosymetric nanocrystals was characterized by using polarized non-linear microscopy techniques that combines two-photon fluorescence and second harmonic generation [9]. Nevertheless, in all these previous works, it was impossible to determine directly the crystal structure of these organic nanocrystals in sol-gel matrices because of their small size and their confinement in amorphous sol-gel matrices. Indeed, X-ray diffraction peaks are enlarged by the nanometer-sized particles. In addition, the low contrast of electronic density between nanocrystals and silicate matrices does not allow extracting the diffraction peaks from the diffusion ring of the amorphous silicate matrix. For this reason, we undertook electron diffraction experiments.

Diffraction patterns were recorded with a CCD camera with a distance camera-sample of $450 \mathrm{~mm}$. Due to sample fragility, TEM observations were carried out at $-173^{\circ} \mathrm{C}$ in liquid nitrogen cooled holder to reduce the detrimental effect of beam damage. This procedure allowed adjusting the focus conditions at an area nearby the region of interest to prevent any unnecessary irradiation prior to record nanocrystal images and diffraction patterns. Thus, we have observed both tetracene free microcrystals and tetracene nanocrystals embedded in sol-gel thin films as shown in Figure 4.

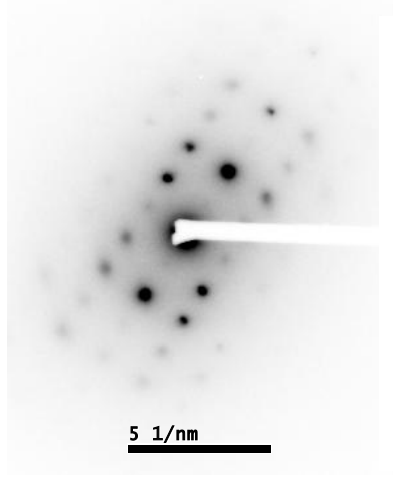

$4 a$

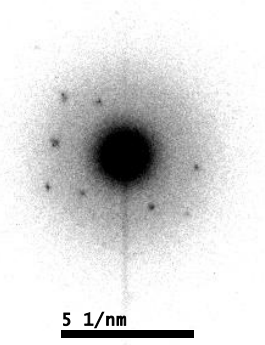

$4 \mathrm{~b}$

Figure 4 : Diffraction patterns obtained for tetracene free microcrystals (a) and tetracene nanocrystals embedded in a sol-gel thin film (b). 
Figures $4 \mathrm{a}$ and $4 \mathrm{~b}$ show respectively the diffraction patterns obtained for tetracene free microcrystals and for tetracene nanocrystals in a sol-gel thin film. The diffraction patterns are constituted of sharp diffraction peaks, which evidences a good crystallinity for tetracene microcrystals and nanocrystals. We can observe, in both cases, the same symmetry of diffraction patterns. This proves that tetracene nanocrystals grown in sol-gel coatings exhibit the same crystal structure to that obtained for free microcrystals crystallized from solutions. An overview was done on tetracene crystalline structure with the Cambridge Structural Database. All the previous structural studies have identified for tetracene a triclinic crystalline system, with a $P \overline{1}$ space group [10-14]. X-ray diffraction allowed us to study the structure of tetracene powders in different recrystallization conditions. The $P \overline{1}$ space group was always observed. Then, a simulation of triclinic $P \overline{1}$ diffraction picture was made with JEMS software and leads to the diffraction picture we obtained. Thus, the triclinic $\overline{P 1}$ crystalline system was identified on both the two zone axis orientations of microcrystals and nanocrystals diffraction patterns. In agreement with previous work, this molecule crystallizes with no polymorphism.

\section{CHEMICAL SENSOR PROPERTIES}

The fluorescence extinction of nanocrystals by molecular probes was characterized by time-resolved fluorescence spectroscopy, with a laser excitation at $\lambda=495 \mathrm{~nm}$. Indeed, the information transduction from adsorbed molecular probe to nanocrystals is carried through an energy transfer of excitation around the nanocrystal towards the surface where it jumps to the non fluorescent indicator depending on its absorption band. So the energy transfer modifies the fluorescence lifetime of the nanocrystals. This fluorescence "quenching" is observed on the fluorescence decay of nanocrystals in presence of a molecular probe so depending on the chemical or biological environment. We have used as molecular probe the Cibacron Blue (CB), a typical coloured indicator dissolved at a $1 \mathrm{mmol} / \mathrm{L}$ concentration in aqueous solutions. One drop $(35 \mu \mathrm{L})$ of this solution was then added onto sol-gel coatings to allow the diffusion of the molecular probe towards the nanocrystals. The efficiency of fluorescence quenching depends on several parameters. In this study, we focused on the effect of fluorescent nanoparticles size.

In section 3, we observed by FCM rubrene nanocrystals of $300 \mathrm{~nm}$ and $150 \mathrm{~nm}$ inside the same sol-gel matrix (TMSE + 2 TMOS). Figure 5 shows the fluorescence decays of these nanocrystals with addition of water and $1 \mathrm{mmol} / \mathrm{L} \mathrm{CB}$ aqueous solution.

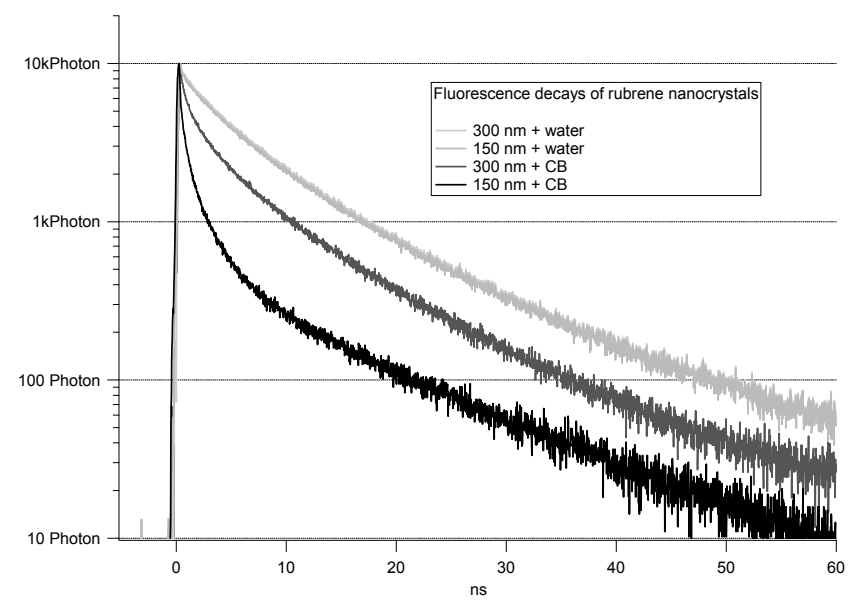

Figure 5 : Fluorescence decays obtained for rubrene nanocrystals of $300 \mathrm{~nm}$ and with addition of water or $\mathrm{CB}$ at $1 \mathrm{mmol} / \mathrm{L}$

In presence of pure water, the same fluorescent decays are observed for nanocrystals of 300 and $150 \mathrm{~nm}$ in diameter. However, in presence of $\mathrm{CB}$, the quenching of luminescence is more important for $150 \mathrm{~nm}$ - nanocrystals than for 300 
$\mathrm{nm}-$ nanocrystals. The decays were then fitted by a biexponential function: $I(t)=A_{1} \exp \left(-\frac{t}{\tau_{1}}\right)+A_{2} \exp \left(-\frac{t}{\tau_{2}}\right)$. Fit results are presented in Table 1.

\begin{tabular}{ccccc}
\hline Decay & $\tau_{1}$ & $\tau_{2}$ & $\% \tau_{1}=\frac{A_{1}}{A_{1}+A_{2}}$ & $\% \tau_{2}=\frac{A_{2}}{A_{1}+A_{2}}$ \\
\hline $\begin{array}{c}300 \mathrm{~nm}+ \\
\text { water }\end{array}$ & $10.36 \mathrm{~ns}$ & $3.25 \mathrm{~ns}$ & $52.6 \%$ & $47.4 \%$ \\
\hline $\begin{array}{c}150 \mathrm{~nm}+ \\
\text { water }\end{array}$ & $10.36 \mathrm{~ns}$ & $3.24 \mathrm{~ns}$ & $52.5 \%$ & $47.5 \%$ \\
\hline $\begin{array}{c}300 \mathrm{~nm}+ \\
\mathrm{CB}\end{array}$ & $10.36 \mathrm{~ns}$ & $2.25 \mathrm{~ns}$ & $36.1 \%$ & $63.9 \%$ \\
\hline $\begin{array}{c}150 \mathrm{~nm}+ \\
\mathrm{CB}\end{array}$ & $10.36 \mathrm{~ns}$ & $1.38 \mathrm{~ns}$ & $14.3 \%$ & $85.7 \%$ \\
\hline
\end{tabular}

Table 1 : Biexponential fits obtained for the decays displayed in Figure 5

Decays obtained in presence of water were exactly the same and could be fitted by a biexponential function with around $52 \%$ of a long fluorescence lifetime of $10.36 \mathrm{~ns}$. Indeed, no energy transfer occurs between the rubrene nanocrystals and the water molecule, because water is not the probe associated to the fluorescent molecule. The short component of fluorescence lifetime, $\tau_{2}$, can be associated to impurities into the initial organic powder or to rubrene molecules located at the surface of the nanocrystal which are much more exposed to incident laser irradiation and to the chemical surrounding (matrix, solution).

To fit decays in presence of $\mathrm{CB}$, the long lifetime $\tau_{1}$ was fixed to the same value obtained for nanocrystals in presence of water, according to the following hypothesis. Energy transfer between a fluorescent molecule and a molecular probe is efficient only on a short distance of around $5 \mathrm{~nm}$, the Förster radius [15]. Therefore, we supposed that nanocrystals were composed of two populations of fluorescent molecules:

-a population situated into the core of the nanocrystals where energy transfer does not happen; these molecules have a long fluorescence lifetime $\tau_{1}$.

-a population situated close to the surface shell of the nanocrystals where energy transfer happens; these molecules have a short fluorescence lifetime $\tau_{2}$.

So the core molecules are not at all concerned by energy transfer so they keep the same fluorescence lifetime under water or CB. But the percentage of this lifetime decreases respectively to $36.1 \%$ and $14.3 \%$ for $300 \mathrm{~nm}$ and $150 \mathrm{~nm}$ nanocrystals. Indeed, when the size of the nanocrystal decrease, the surface/core ratio is enhanced so the percentage of molecules concerned by energy transfer is more important in the smallest nanocrystals. Therefore, to improve the sensitivity of our nanosensors, we have to synthesize nanocrystals of around $100 \mathrm{~nm}$ of diameter.

In this hypothesis, we considered only the direct Förster energy transfer, which implies that excitation is localised on a short distance inside the nanocrystal (typically $5 \mathrm{~nm}$ ). However, a nanocrystal is an aggregation of about $10^{6}$ fluorescent molecules organized in a crystalline network. Thus, the excitation could be spread on a longer distance, because of partial delocalization of the excitation in the volume of the nanocrystal.

\section{CONCLUSION}

This research designed a new type of hybrid organic-inorganic materials through a simple and generic preparation of organic nanocrystals grown in sol-gel matrices. This flexible process was based on the confinement in time of the nucleation while the nanocrystal growth was controlled by the spatial confinement in the pores of the sol-gel network. We compared this method with microcrystallization in free solutions. Whereas microcrystals presented a large size distribution, spherical nanocrystals with narrow size distribution were obtained in thin films nanocomposites through the high control of the matrix polycondensation, the confined nucleation and the low diffusion coefficients in the viscous sol. We also greatly improved the stability (mechanical, thermal, chemical and photochemical) of molecular nanocrystals through their strong confinement in pores of dense sol-gel matrices. We showed that the size of the 
nanocrystals could be adjusted through the variation of dye concentration $\mathrm{d}$. Then, electron diffraction was performed on tetracene free microcrystals and tetracene nanocrystals in sol-gel thin films. A comparison between the two samples allowed us to prove that these nanoparticles were crystallized in a triclinic crystalline system with a $P \overline{1}$ space group. Finally, time-resolved fluorescence spectroscopy was performed to evidence chemical sensor properties of the nanocrystals. We have synthesized both $150 \mathrm{~nm}$ and $300 \mathrm{~nm}$ nanocrystals in diameters. The addition of a molecular probe $(\mathrm{CB})$ on these two sizes of nanocrystals showed that $\mathrm{CB}$ quenching of the luminescence is more efficient for 150 $\mathrm{nm}$ sized nanocrystals. Indeed, in smaller nanocrystals the surface/volume ratio is enhanced, which favors the transfer of the excitation in a larger part of the nanocrystal. Works are now in progress to open significantly the pore size of the nanocrystals matrices to optimize the diffusion of chemical or biological probes towards the nanocrystals.

\section{REFERENCES}

[1] A. Ibanez, S. Maximov, A. C. Guiu, C. Chaillout, P. L. Baldeck, "Controlled Nanocrystallization of Organic Molecules in Sol-Gel Glasses", Adv. Mater., 10, 1540-1543, 1998

[2] N. Sanz, A. C. Gaillot, P. L. Baldeck, A. Ibanez, "Organic nanocrystals grown in sol-gel coating", J. Mater. Chem., 10, 2723, 2000

[3] J. Zaccaro, N. Sanz, E. Botzung-Appert, P. L. Baldeck, A. Ibanez, "Organic nanocrystals grown in sol-gel matrices: a new type of hybrid material for optics", C. R. Phys., 3, 463-478, 2002

[4] B. Boury, R. J. P. Corriu, "Auto-organisation of hybrid organic-inorganic materials prepared by sol-gel process", Chem. Comm., , 795-802, 2002

[5] V. K. LaMer, R. H. Dinegar, "Theory, Production and Mechanism of Formation of Monodispersed Hydrosols", J. Am. Chem. Soc., 72, 4847-4854, 1950

[6] V. Monnier, N. Sanz, E. Botzung-Appert, M. Bacia, A. Ibanez, "Confined nucleation and growth of organic nanocrystals in sol-gel matrices", J. Mater. Chem., 16, 1-11, 2006

[7] N. Sanz, A. Boudet, A. Ibanez, "Melting Behavior of Organic Nanocrystals Grown in Sol-Gel Matrices", J. Nanoparticle Res., 4, 99-105, 2002

[8] N. Sanz, P. L. Baldeck, J. F. Nicoud, Y. Le Fur, A. Ibanez, "Polymorphism and luminescence properties of CMONS organic crystals: bulk crystals and nanocrystals confined in gel-glasses", Solid State Sciences, 3, 867875,2001

[9] S. Brasselet, V. LevFloc'h, F. Treussart, J. F. Roch, J. Zyss, E. Botzung, A. Ibanez, "In Situ Diagnostics of the Crystalline Nature of Single Organic Nanocrystals by Nonlinear Microscopy", Phys. Rev. Lett., 92, 207401207402, 2004

[10] R. B. Campbell, J. M. Robertson, "The crystal structure of hexacene, and a revision of the crystallographic data for tetracene and pentacene", Acta Cryst., 15, 289, 1962

[11] E. Eiermann, G. M. Parkinson, H. Bässler, J. M. Thomas, "Structural Investigations of Amorphous Tetracene and Pentacene by Low-Temperature Electron Diffraction", J. Phys. Chem., 87, 544-551, 1983 
[12] E. Hertel, H. W. Bergk, "Der strukturelle Feinbau von kondensierten aromatischen Kohlen wasserstoffen und ihren Molekülverbindungen mit Trinitrobenzol", Z. Physikal. Chem. B, 33, 319-333, 1936

[13] D. Holmes, S. Kumaraswamy, A. J. Matzger, K. P. C. Vollhardt, "On the Nature and Nonplanarity in the [N]Phenylenes", Chem. Eur. J., 11, 3399-3412, 1999

[14] J. M. Robertson, V. C. Sinclair, J. Trotter, "The Crystal and Molecular Structure of Tetracene", Acta Cryst., 14, 697-704, 1961

[15] T. Förster, Light and Organic Crystals: delocalized excitation and excitation transfer. 1965: Academic Press: New York.

*virginie.monnier@grenoble.cnrs.fr; phone 00334768878 11; fax 0033476881038 